\title{
Catastrophes and Chaos in Business Cycle Theory
}

\author{
A. JAKIMOWICZ \\ The University of Economy, Garbary 2, PL-85-229 Bydgoszcz, Poland
}

\begin{abstract}
The primary thesis of this paper is that a nonlinear dynamical systems theory provides a basis for conducting all kinds of comparisons in the theory of business cycles, and it also enables its further development. A cognitive aim was to show that applying the theory of bifurcations and morphogenesis in the domain of economic fluctuations allows us to construct models of the cycle with greater explicatory and utility values than there were so far. In this way, the precision and consistency of the theory increases. In this field, applications of catastrophe theory are of great importance. Another fact was indicated, namely the theory of deterministic chaos places the issues of explanation and forecasting in economics in a totally different light. It turns out that we are dealing with at least three sources of complexity in economic systems: chaotic attractors, invariant chaotic sets that are not attracting in the form of chaotic saddles and the effects of fractal basin boundaries. This, in turn, limits the effectiveness of traditional economic policy. Economic management should be based on procedures that lower complexity of economic systems, however sometimes lower complexity incurs bigger instability. The paper is a survey of applications of nonlinear dynamical systems to mathematical business cycle models. The survey encompasses both earlier models that were built in 1970s, as well as later concepts. The paper also features a few of my newest results of numerical studies of some nonlinear economic systems.
\end{abstract}

PACS numbers: 05.45.-a, 89.20.-a, 89.65.Gh, 89.75.-k

\section{Introduction}

The catastrophe theory, known also as the theory of morphogenesis, was developed by a French mathematician Thom [1] in the 1960s. It is a general method of systems modeling, which can explain in particular how discontinuous effects come to being from continuous causes. At first, it was used to describe a biological morphogenesis, however it was relatively rapidly applied in other walks of science, mainly in physics [2-4]. The main mathematical source for this method is topology. Owing to this, the catastrophe theory appeals to geometrical intuition of a researcher.

Let the dynamical system be described with a smooth function:

$$
f: \mathbb{R}^{k} \times \mathbb{R}^{n} \rightarrow \mathbb{R},
$$

with control space $\mathbb{R}^{k}$ (causes) and state space $\mathbb{R}^{n}$ (effects). It is most often assumed that $k \leq 5$, but there are no limits regarding $n$. The function (1) can represent potential, energy, probability, or cost. The last interpretation is particularly valuable from an economic point of view, because it naturally confirms that a basic law of economics is observed in the systems - the rational managing principle.

Catastrophe means violent, sudden transition of the tested system into a new state. It does not necessarily have to be a state worse than its predecessor, so the term "catastrophe" has a much broader meaning in this theory than the colloquial one, where it is associated with something evil or hazardous, e.g. some kind of disaster. Catastrophe is illustrated by boiling water in a kettle, a bull market, or a company going into liquidation. What is important here is the rapidity of changes in the behavior of an object as compared with the mean change in the past. The catastrophe theory merges two apparently contradictory and unrelated kinds of phenomena descriptions to form one coherent notion system: evolutionism and revolutionism, continuity and discontinuity. It allows us to present a complete way of an object as continuous changes, which are interrupted by sudden quality changes. In this way, this theory becomes a useful tool in structural research.

The classification theorem forms a basis for the catastrophe theory. According to it the number of elementary catastrophes, i.e. all possible ways of manifesting discontinuity, is finite if the dimension of control space does not exceed five. Above this value the classification becomes infinite. For a dimension equal to five we acquire eleven elementary catastrophes, which are further divided into cuspoids represented by functions of one variable and umbilics represented by functions of two variables. There are five types of cuspoids: fold, cusp, swallowtail, butterfly and wigwam. In addition, there are six types of umbilics: hyperbolic, elliptic, parabolic, second hyperbolic, second elliptic, and symbolic. The names of elementary catastrophes come from similarity that can be observed between geometry of singularities and commonly known shapes. They are represented with simple polynomials, hence the catastrophe theory is a method available not only for mathematicians, although it is relatively difficult to prove the classification theorem [5].

The application of the catastrophe theory in economics is feasible only when we use relatively strong scientific hypotheses as our basis. They do not have to be quan- 
tified. If it is impossible to know what factors are key in making investment decisions or specifying the amount of government spending, the catastrophe theory is futile. The mathematical theorem itself does not yield economic laws or any other, but it can help identify or specify them. No sooner has the hypothesis describing a given phenomenon or process been initially formed, than we can expect the catastrophe theory to facilitate forming the simplest mathematical structure that will generate system behavior close to the one we can observe in reality. The acquired models of phenomena can possess some predictive properties, obviously within the scope of predictability horizon determined by the theory of deterministic chaos. The limits of predictability of social phenomena with nonlinear models have been discussed by A. Saperstein [6], especially in the arms race context.

In order for the catastrophe theory to be implemented, the tested systems have to reveal certain quality features. These are as follows:

- Discontinuity which is present when a system behavior set breaks into types of various quality, and the jumps between them take place as a result of continuous changes in the causes set.

- Divergence which means that big changes in the state space of a given system are a result of a insignificant shift in the trajectory in parameter space, and the discontinuous change (catastrophe) does not take place.

- Multimodality which means that the investigated system has more than one stable equilibrium state.

- Alternativity that is present when the transition between two points in the parameter space can take place due to both continuous changes and discontinuous shifts.

- Inaccessibility. The catastrophe theory describes states that are most probable, and it omits all the rest.

It is possible to apply the chaos theory in economics, because economic laws can be written as nonlinear differential or difference equations, the control parameters are managed from outside, and the tested systems have more than two degrees of freedom. In these conditions it is quite common to observe sensitive dependence on initial conditions (butterfly effect), which on the one hand limits the predictability horizon and on the other it enables a modification of chaotic states.

It is often presumed that the role of nonlinear models merely comes down to enhancement of epistemological value of hitherto existing mathematical economic considerations. This viewpoint is true, but only to some extent. Over the last couple of years, application of nonlinear dynamical systems and agent-based computational models has contributed to an emergence of a new science called complexity economics. It means radical remaking of traditional economics. The most important discrepancies concern such basic issues as dynamics, agents, networks, emergence, evolution, technology, preferences, origins, elements and the time horizon of forecasts [7-10]. It was mainly a group of scientists associated with Santa Fe Institute, Gell-Mann [11], Brian Arthur [12, 13], Holland $[14,15]$ and Epstein [16, 17], who were the precursors of this science. The concept of general equilibrium, which constitutes a basis for mainstream economics, does not necessarily have to be rejected since it is correct in describing certain types of behavior; however, these are merely extraordinary states of systems. Thus, it should be extended with a new basis for economic reasoning - nonlinear dynamics. This is a creed of complexity economics; its purpose is to generalize standard economics [18]. However, it is a revolutionary generalization of the Keynesian economics kind.

The road to complexity economics leads through proper application of metaphors. The significance of metaphors in economics and organization science has been widely discussed by Mirowski [19, 20] and Mesjasz [21]. From an epistemological point of view the most efficient method of building nonlinear mathematical models of economic phenomena is to treat ideas taken from natural sciences as foundations (source fields) of analogies and metaphors. If this kind of metaphoric thinking can be subject to mathematization, what we get is the progress factor in economics and other soft (social) sciences.

Nonlinear models, as all other models, are subject to some limitations, they are a simplified reflection of reality. Thus they cannot provide a complete explanation of many complex economic phenomena. They are a mere approximation but this approximation is better than the one provided by standard models. Usefulness of nonlinear models grows when we apply them to describe such phenomena where parameters can be precisely defined. In particular it concerns models of financial markets.

New economics can lead to many surprising discoveries: it can help explain the causes of the fall of socialist economy, diagnose some dangers in the process of transformation or identify new sources of economic complexity. What is important, the mentioned phenomena generate social costs, which are usually ignored in economic calculations.

\section{Applications of catastrophe theory in economics}

In the middle of 1970 s the catastrophe theory became trendy with scientific circles, which resulted in more or less successful applications in social sciences [22-24]. To a large extent it was caused by some dramatic political and economic events of that period, such as the first oil shock and the corresponding intellectual atmosphere that was spawned by it. At the same time, there has been some high expectations evoked concerning working 
out a scientific theory that could describe quality and structural changes within systems that have focused on the catastrophe theory. It was mainly due to Zeeman who transformed it to a form that proved helpful in applications, and he provided many benchmark examples of its usage. A few years later, it met with a wave of criticism [25-29], which is not always deemed justified today, however as a result, there were fewer and fewer original applications of the catastrophe theory in the following periods. Besides, economics experienced fascination and a drop in interest in the catastrophe theory.

Currently, it is often thought that the catastrophe theory has a rather limited application in modeling economic phenomena. Most often it is justified by the fact that variables present in some catastrophe models are mere metaphors, linguistic variables, which do not have any precise significance. Social phenomena described by so broadly-defined categories are inherently nonlinear and can be interpreted by various models, and the catastrophe theory is just one of the possibilities. It happens very often. This circumstance might indeed discourage one to do research in this direction. However, such approach seems to be too biased. Efficacy of such a method lies in the fact that elementary catastrophes can be represented by simple polynomials, hence application of the catastrophe theory allows us to formulate the simplest mathematical model that approximates the explored phenomena. In such a case it is advisable to use this method at least in the preliminary stage of modeling. In further stages other methods can be taken into consideration. We can start with the catastrophe theory, but it does not necessarily mean that we have to finish with it. Modeling is a continuous process with a view to achieve ever better approximations of actual phenomena. Structures that resemble elementary catastrophes tend to recur in many advanced applications, which makes us presume that there are still best things to come for this method in the future. Moreover, in catastrophe models we can find typical economic variables such as inflation, unemployment or economic growth, and those notions are well defined. In such cases the achieved models are usually immediately better than their linear equivalents that are provided by conventional economics.

In the 1970s the catastrophe theory became an important factor in the development of theoretical explorations. The most important achievement was the fact that it introduced complexity into economics. Elementary catastrophes were the first novel form of nonlinear, topological complexity which was seriously studied in economics $[30,31]$. It enabled economists to make research more realistic and to step beyond an analysis of equilibrium states that emerged from linear models. Simultaneously, the first applications of the catastrophe theory provided arguments that questioned the theory of rational expectations, which was at that time a basic exploratory assumption. Zeeman [32] worked out a model of securities market based on the cusp catastrophe, which assumed the existence of two kinds of players, fundamentalists or chartists. It did not fit with the image of rational agents.
In that period these features of a model were subject to severe criticism. Today, however, it is different, heterogeneity of agents is one of the basic features of many market and economy models.

Occasionally critics claim that although catastrophe models formulated in social sciences are usually elegant from a formal point of view, serious problems occur when trying to estimate their parameters. It is true to a large extent. On the one hand, it is caused by the lack of proper statistical procedures, on the other - insufficient mathematical specification of models. Solving these problems could constitute one of potential research directions in this field. Recently, some progress has been recorded, an example of which could be empirical methods such as multi-modal models. As far as economics is concerned, the first step has already been taken by Fischer and Jammernegg [33], who carried out an estimation of catastrophic unfolding of the Phillips curve and they acquired results that confirm its great usefulness in comparison with the traditional approach.

Ultimately it turned out that the presented perturbations did not diminish by any means the explicatory values of catastrophe theory, due to which it constitutes an integral part of economic methodology, and the relatively long period that has passed since its first applications enables us to correctly estimate not only the possibilities it offers but also its limitations. According to the Hegel observation, the Minerva owl flies out at dusk. Rosser [34] assesses the situation that emerged due to applications of the catastrophe theory in economics as follows:

In sum, it would appear that indeed the baby
of catastrophe theory was largely thrown out
with the bathwater of its inappropriate ap-
plications to a large extent. Although there
are serious limits to its proper application in
economics, there remain many potential such
proper applications. Economists should no
longer shy away from its use and should in-
clude it with the family of other methods for
studying dynamic discontinuity. It should be
revalued from its currently low state on the
intellectual bourse and right the wrong of its
excessive devaluation, while avoiding any re-
turn to the hype and overvaluation that oc-
curred during the 1970s. A reasonable middle
ground can and should be found.

Discontinuous transitions that are most often found in applications include the cusp catastrophe and the butterfly catastrophe. They are both featured in Table. The dimension of state space in both cases is the same and it is equal to one, whereas the dimension of control space amounts to two in the case of the cusp and four in the case of the butterfly. The polynomial coefficients have been selected in such a way as to simplify the definitions of catastrophe manifolds, the sets of singularities and the sets of bifurcations. 
TABLE

Features of the cusp catastrophe and the butterfly catastrophe.

\begin{tabular}{|c|c|c|}
\hline $\begin{array}{c}\text { Basic } \\
\text { characteristics }\end{array}$ & Cusp catastrophe & Butterfly catastrophe \\
\hline $\begin{array}{l}\text { dimension of } \\
\text { state space }\end{array}$ & 1 & 1 \\
\hline $\begin{array}{l}\text { dimension of } \\
\text { control space }\end{array}$ & 2 & 4 \\
\hline representation & $f: \mathbb{R}^{2} \times \mathbb{R}^{1} \rightarrow \mathbb{R}$ & $f: \mathbb{R}^{4} \times \mathbb{R}^{1} \rightarrow \mathbb{R}$ \\
\hline $\begin{array}{l}\text { potential } \\
\text { function }\end{array}$ & $f(a, b, x)=\frac{1}{4} x^{4}+\frac{1}{2} b x^{2}+a x$ & $\begin{array}{c}f(a, b, c, d, x)=\frac{1}{6} x^{6}-\frac{1}{4} d x^{4} \\
-\frac{1}{3} c x^{3}-\frac{1}{2} b x^{2}-a x\end{array}$ \\
\hline manifold & $\begin{aligned} M_{3} & =\left[(a, b, x): \frac{\mathrm{d} f}{\mathrm{~d} x}=0,\right. \\
\frac{\mathrm{d} f}{\mathrm{~d} x} & \left.=x^{3}+b x+a=0\right]\end{aligned}$ & $\begin{array}{c}M_{5}=\left[(a, b, c, d, x): \frac{\mathrm{d} f}{\mathrm{~d} x}=0\right. \\
\left.\frac{\mathrm{d} f}{\mathrm{~d} x}=x^{5}-d x^{3}-c x^{2}-b x-a=0\right]\end{array}$ \\
\hline singularity set & $\begin{array}{l}S_{3}=\left[(a, b, x): \frac{\mathrm{d}^{2} f}{\mathrm{~d} x^{2}}=0,\right. \\
\left.\quad \frac{\mathrm{d}^{2} f}{\mathrm{~d} x^{2}}=3 x^{2}+b=0\right]\end{array}$ & $\begin{array}{c}S_{5}=\left[(a, b, c, d, x): \frac{\mathrm{d}^{2} f}{\mathrm{~d} x^{2}}=0\right. \\
\left.\frac{\mathrm{d}^{2} f}{\mathrm{~d} x^{2}}=5 x^{4}-3 d x^{2}-2 c x-b=0\right]\end{array}$ \\
\hline bifurcation set & $B_{3}=\left[(a, b): 4 b^{3}+27 a^{2}=0\right]$ & $B_{5}=[(a, b, c, d): F(a, b, c, d)=0]$ \\
\hline
\end{tabular}

Exemplary models of business cycles can be as follows:

$$
\left.\begin{array}{l}
\text { Balance of payments } \\
\text { Unemployment }
\end{array}\right\} \begin{aligned}
& \text { economic policy } \\
& \text { (for the cusp catastrophe). }
\end{aligned}
$$

Or

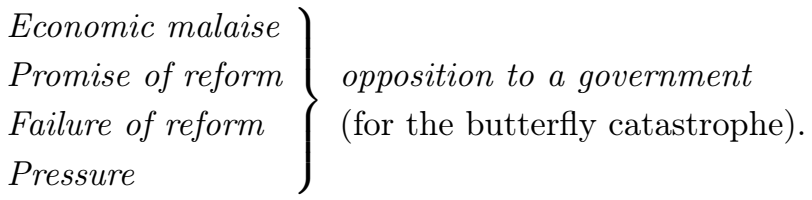

More details can be found in [35-38]. Elementary catastrophes enable us to create in a simple way a mathematical representation of quality economic phenomena, which is difficult to achieve in other ways.

\section{Deterministic chaos in macroeconomics}

\subsection{Actual causes of the fall of the centrally planned economy}

One of the most interesting models of the functioning of real socialism comes as two-dimensional piecewise linear map [39, 40]:

$$
\begin{aligned}
& e_{t}=f e_{t-1}+\sigma_{s} s\left(e_{t-1}, a_{t-1}\right)-i\left(e_{t-1}\right)-\varepsilon_{0}, \\
& a_{t}=\beta_{i} i\left(e_{t-1}\right)-\beta_{0}
\end{aligned}
$$

$$
\begin{aligned}
& s\left(e_{t-1}, a_{t-1}\right)=\left\{\begin{array}{l}
s^{l} \\
\sigma-\sigma_{e} e_{t-1}-\sigma_{a} a_{t-1} \\
s^{u}
\end{array},\right. \\
& i\left(e_{t-1}\right)=\left\{\begin{array}{l}
i^{l} \\
\iota+\iota_{e} e_{t-1} . \\
i^{u}
\end{array}\right.
\end{aligned}
$$

The stage variables are: $e$ - internal tension, $a-$ external tension. The remaining symbols determine parameters. The parameter space is 15-dimensional.

The internal tension is the deviation of the commitment ratio from its minimum value. The commitment is the expected remaining costs of the investment projects under construction at the end of a year $t$. The external tension is the deviation of the net import ratio from its minimum value, which depends on the structure of foreign trade of a socialist economy. The notions of internal and external tensions can be applied only to a socialist economy; they are useless in the case of a capitalist economy.

The most frequent dynamic changes in the model (2)-(5) are border-collision bifurcations. They result from Hicksian nonlinearities [41]. Border-collision bifurcations are related to closed invariant sets having a contact with the border of a region of the map's definition. Figure 1 features one of the results I acquired when I carried out numerical explorations of Simonovits' model. A few border-collision bifurcations can be identified for the growing bifurcation parameter: fixed point attrac- 


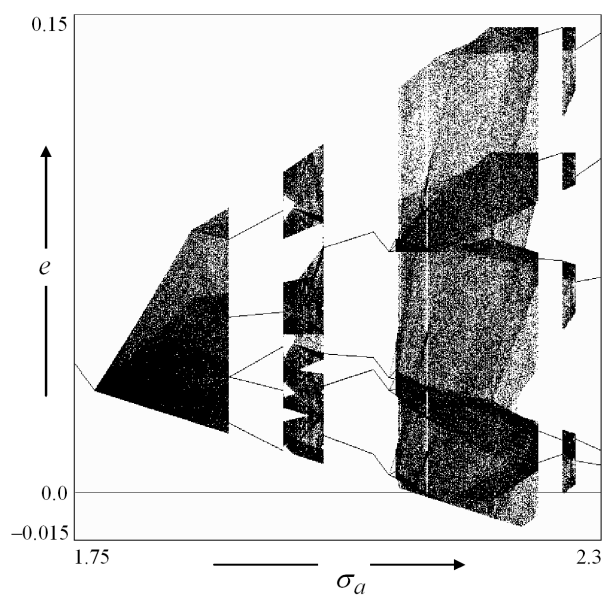

Fig. 1. The bifurcation diagram of the internal tension. It shows border-collision bifurcations which stem from Hicksian nonlinearity.

tor $\rightarrow$ chaotic attractor, chaotic attractor $\rightarrow$ period 5, chaotic attractor $\rightarrow$ period 4 , period $4 \rightarrow$ period 12 , period $12 \rightarrow$ chaotic attractor. This indicates impulsive and unpredictable changes in economic complexity. The jumps between the states of various complexity come as a complete surprise to a central planner, which makes it impossible to pursue economic policy effectively. If the system goes into the state of chaos, its further existence is jeopardized. In a socialist economy, economists did not consider the costs relating to changes in economic complexity, hence that type of economy was doomed.

\subsection{Economic transformation: a trade-off between complexity and instability}

The starting point for the nonlinear system transformation theory can be the concept worked out by a team of American researchers, which was tested on the basis of Russian economy data $[42,43]$. It is based on a simultaneous application of two methods: the chaos theory and the catastrophe theory. The basic feature of this model is a chaotic hysteresis, which is a result of a two-stage nonlinear accelerator that generates an investment cycle. In order to describe socio-economic crises the notion of a technological gap and the cusp catastrophe were used. In this way, a concise picture of the transformation process is obtained covering the phenomenon of hysteresis, chaotic dynamics, and non-continuous transitions.

A two-stage accelerator was defined as follows [44]:

$$
\begin{aligned}
& I_{t}=I_{t-1}+Z_{t}, \\
& Z_{t}=u\left(Z_{t-1}-Z_{t-1}^{3}\right)-v I_{t-1},
\end{aligned}
$$

where: $I-$ stands for total investment, $Z$ - stands for growth in investment, whereas $u$ and $v$ represent parameters. If the value of the accelerator operating in the capital goods sector is set at $u=2$, and then the consumer goods accelerator $v$ is reduced, the chaotic hysteresis process will be observed. Low values of the parameter $v$ mean putting emphasis on investment in the consumer goods industry and they help reduce chaos in the system.

Model (2)-(5) shows that the basic problem of a socialist economy lies in the investment cycle in the form of a chaotic attractor, thus any reform should start with endeavors to lower the complexity of economy. In the system (6)-(7), it can be achieved through lowering the $v$ parameter. However, as we reduce the system's complexity it becomes more unstable. I called this phenomenon a trade-off between complexity and instability [45]. Reducing complexity increases instability, and decreasing instability increases complexity, which is represented by an increase in Lyapunov exponents of other attractors and an increase in their box, Lyapunov and correlation dimensions.

\subsection{New sources of economic complexity: chaotic saddles and the effects of fractal basin boundaries}

A key element in the contemporary theory of business cycle is the Goodwin model from 1951 [46]. In the subject's literature it is written down as follows:

$$
\frac{\mathrm{d}^{2} x}{\mathrm{~d} t^{2}}+a \frac{x^{2}-1}{x^{2}+1} \frac{\mathrm{d} x}{\mathrm{~d} t}-b x+c x^{3}=d \sin (\omega t),
$$

where the variable $x$ stands for deviations of national income from the equilibrium, whereas $a, b, c, d, \omega$ are parameters. Equation (8) describes the forced oscillator, which is one of the most interesting nonlinear dynamical systems in science.

An important property of the Goodwin model are the chaotic saddles, which can be situated within the basins of attraction of periodic attractors. Chaotic saddle is an invariant compact set $C$ that is neither attracting nor repelling, and there exists a point in $C$ whose trajectory is chaotic and travels throughout $C$. Straddle methods are techniques for observing trajectories that lie in invariant sets. Straddle trajectory is a sequence of short line segments and each of the segments must straddle a specified set $S$ [47].

Subsequently, we computed the saddle straddle trajectory (SST) in the Goodwin model, which is shown in Fig. 2 [48]. In this case, the parameter values are as follows: $a=0.8, b=0.6, c=0.5, d=9.5$ and $\omega=1$. SST is in the basin of attraction of the period 3 attractor. There exists a chaotic saddle in the phase space of the system. The number of the plotted points of the trajectory is $10^{6}$. The Lyapunov exponents of this trajectory are $\lambda_{1}=0.153358, \lambda_{2}=-0.400679$. The estimate for the capacity dimension of the chaotic saddle is $1.155 \pm 0.061$ and the estimate for the correlation dimension is $1.076 \pm 0.055$. Lorenz and Nusse [49] noticed that there exists a similar chaotic saddle in the basin of attraction of a periodic attractor of period 3 for $a=0.75$, $b=c=0.5, d=10$ and $\omega=1$. Thus, the saddle does not disappear even if there are some small shifts in parameters.

The occurrence of chaotic saddles leads to transient chaotic behavior, although there is a periodic attractor of period 3 in the phase space. This type of behavior is 


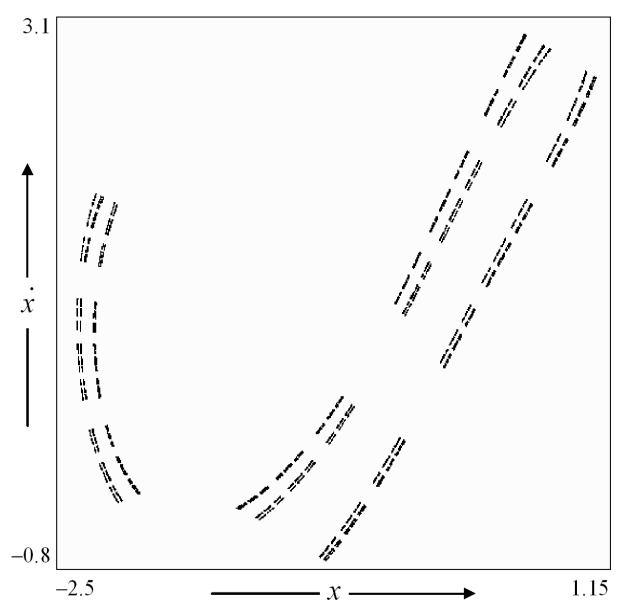

Fig. 2. The chaotic saddle is the compact invariant (Cantor-like) set which is responsible for the transient chaotic dynamics.

very important from an economic point of view, because it accounts for complex behavior of economy even if the chaotic attractor is missing.

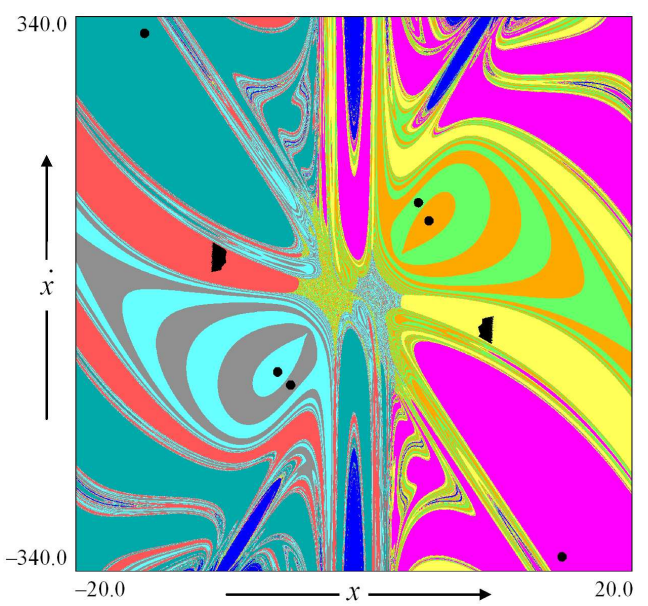

Fig. 3. The phase space of the Goodwin model with basins of attraction of nine attractors: the point at infinity, six stable fixed points, and two chaotic attractors.

Precise numerical explorations of the Goodwin model lead me to a discovery that fractal basin boundaries can be another source for economic complexity. Figure 3 shows a fragment of the phase space of this system. The accepted parameters values are as follows: $a=43.3$, $b=c=0.5, d=27.4, \omega=1$. The fragment of the phase space contains nine co-existing attractors together with correlating attraction sets. There are six attracting stable points that are symbolically marked with black circles, and two chaotic attractors. The ninth attractor is the point at infinity. Occurrence of many different attractors in a dynamic system brings about serious consequences for global dynamics. If there was even a small external noise in the system, then - due to a tan- gled structure of these basins - it could influence the initial conditions and move them between different regions of phase space, which in turn, could bring about irregular leaps between chaotic attractors or the periodic ones. It will lead to a much more complicated behavior of the system instead of following one of the chaotic attractors, which occupy a specific place within the phase space. A similar phenomenon was discovered in physical systems [50]. Fractal basin boundaries in the Goodwin model show an important source of economic complexity. This notion is only hinted at here and it requires further, more precise explorations.

\section{Summary}

Introducing the catastrophe theory into economics in the middle 1970s was a first serious attempt at exploring macroeconomic complexity, although it questioned a common at that time belief in rational expectations. Today, occurrence of heterogeneous agents is recognized as standard. Nonlinear dynamics should make economists aware that complexity is a basic trait of economies and that it generates costs that have not been included in economic calculations so far. From the point of view of efficiency of economic policy it is especially important to pinpoint sources of economic complexity and working out methods of their control. The sources of complexity lie not only in chaotic attractors, but also in invariant chaotic sets that are not attracting and fractal basin boundaries. What is interesting, we can arrive at these conclusions exploring classic, archetypal economic models, three of which were presented in this article. It means that nonlinear dynamics should be a basic tool for a theoretical economist, because in this way we can reduce a growing gap between claims of economic theory and behavior of actual markets and economies.

\section{References}

[1] R. Thom, Structural Stability and Morphogenesis. An Outline of a General Theory of Models, Benjamin, London 1975.

[2] R. Gilmore, Catastrophe Theory for Scientists and Engineers, Dover Publications, New York 1993.

[3] T. Poston, I. Stewart, Catastrophe Theory and its Applications, Dover Publications, Mineola 1996.

[4] A.E.R. Woodcock, M. Davis, Catastrophe Theory: A Revolutionary Way of Understanding how Things Change, Pelican, London 1980.

[5] D.J.A. Trotman, E.C. Zeeman, in: Structural Stability, the Theory of Catastrophes, and Applications in the Sciences, Ed. P. Hilton, Springer, Berlin 263 (1976).

[6] A.M. Saperstein, in: Chaos Theory in the Social Sciences: Foundations and Applications, Eds. L.D. Kiel, E. Elliott, The University of Michigan Press, Ann Arbor 139 (2004).

[7] E.D. Beinhocker, Origin of Wealth: Evolution, Complexity, and the Radical Remaking of Economics, Harvard Business School Press, Boston 2006. 
[8] The Complexity Vision and the Teaching of Economics, Ed. D.C. Colander, Edward Elgar Publications, Cheltenham 2000.

[9] The Economy as an Evolving Complex System II, Eds. W.B. Arthur, S. Durlauf, D.A. Lane, Addison-Wesley, Reading 1997.

[10] A. Jakimowicz, Ekonomista 1, 15 (2009).

[11] M. Gell-Mann, The Quark and the Jaguar: Adventures in the Simple and the Complex, W.H. Freeman, New York 1994.

[12] W.B. Arthur, Complexity 1, 20 (1995).

[13] W.B. Arthur, Science 284, 107 (1999).

[14] J.H. Holland, Hidden Order: How Adaptation Builds Complexity, Addison-Wesley, New York 1995.

[15] J.H. Holland, Emergence: From Chaos to Order, Oxford University Press, Oxford 1998.

[16] J.M. Epstein, Nonlinear Dynamics, Mathematical Biology, and Social Science, Addison-Wesley, Reading 1997.

[17] J.M. Epstein, Generative Social Science: Studies in Agent-Based Computational Modeling, Princeton University Press, Princeton 2006.

[18] W.B. Arthur, Complexity in the Economy and Business, IBM Almaden Institute, San Jose 2007.

[19] P. Mirowski, More Heat than Light: Economics as Social Physics, Physics as Nature's Economics, Cambridge University Press, Cambridge 1989.

[20] Natural Images in Economic Thought: "Markets Read in Tooth and Claw". Historical Perspectives of Modern Economics. Ed. P. Mirowski, Cambridge University Press, Cambridge 1994.

[21] C. Mesjasz, S.E.E.D. Journal (Semiotics, Evolution, Energy, and Development) 1, No. 2 (2001).

[22] G. Ribeill, Metra 14, 499 (1975).

[23] I. Stewart, New Scientist 68, 447 (1975).

[24] E.C. Zeeman, in: Proceedings of the International Conference on Manifolds and Related Topics in Topology,ed. A. Hattori, University of Tokyo Press, Tokyo 11 (1975).

[25] J.G.A. Croll, New Scientist 70, 630 (1976).

[26] H.J. Sussmann, R.S. Zahler, The Sciences 17, 20 (1977).

[27] H.J. Sussmann, R.S. Zahler, Behavioral Science 23 , 383 (1978).

[28] H.J. Sussmann, R.S. Zahler, Synthese 37, 117 (1978).

[29] R.S. Zahler, H.J. Sussmann, Nature 269, 759 (1977).

[30] J.B. Rosser, Jr., Implications for Teaching Macroeconomics of Complex Dynamics, Department of Economics, James Madison University, Harrisonburg 2004 .
[31] H.W. Gottinger, in: On the Stability of Contemporary Economic Systems, Eds. O. Kýn, W. Schrettl, Vandenhoeck und Ruprecht, Göttingen 422 (1979).

[32] E.C. Zeeman, J. Mathematical Economics 1, 39 (1974).

[33] E.O. Fischer, W. Jammernegg, Review of Economics and Statistics 68, 9 (1986).

[34] J.B. Rosser, Jr., The Rise and Fall of Catastrophe Theory Applications in Economics: Was the Baby Thrown Out with the Bathwater?, Thirteenth Annual Symposium of the Society for Nonlinear Dynamics and Econometrics, City University, London 2005.

[35] A. Jakimowicz, Od Keynesa do teorii chaosu. Ewolucja teorii wahań koniunkturalnych, Wydawnictwo Naukowe PWN, Warszawa 2005 (in Polish).

[36] J.B. Rosser, Jr., From Catastrophe to Chaos: A General Theory of Economic Discontinuities, Kluwer Acad. Publications, Boston 1992.

[37] C.A. Isnard, E.C. Zeeman, in: The Use of Models in the Social Sciences, ed. L. Collins, Tavistock Publications, London 44 (1976).

[38] E.C. Zeeman, Catastrophe Theory: Selected Papers, 1972-1977, Addison-Wesley, London 1977.

[39] A. Simonovits, Economics of Planning 24, 27 (1991).

[40] C.H. Hommes, H.E. Nusse, A. Simonovits, J. Economic Dynamics and Control 19, 155 (1995).

[41] J.R. Hicks, A Contribution to the Theory of the Trade Cycle, Clarendon Press, Oxford 1951.

[42] J.B. Rosser, Jr., M.V. Rosser, Discrete Dynamics in Nature and Society 1, 269 (1998).

[43] J.B. Rosser, Jr., M.V. Rosser, S.J. Guastello, R.W. Bond, Nonlinear Dynamics, Psychology, and Life Sciences 5, 345 (2001).

[44] T. Puu, Annals of Operations Research 37, 169 (1992).

[45] A. Jakimowicz, Nonlinear Dynamics, Psychology, and Life Sciences 13, 393 (2009).

[46] R.M. Goodwin, Econometrica 19, 1 (1951).

[47] H.E. Nusse, J.A. Yorke, Dynamics: Numerical Explorations, Springer, New York 1998

[48] A. Jakimowicz, Nonlinear Dynamics, Psychology, and Life Sciences 14, 69 (2010).

[49] H.-W. Lorenz, H.E. Nusse, Chaos, Solitons, and Fractals 13, 957 (2002).

[50] M. Iansiti, Q. Hu, R.M. Westervelt, M. Tinkham, Phys. Rev. Lett. 55, 746 (1985). 